\title{
On the fragmentalist interpretation of special relativity
}

\author{
Martin A. Lipman ${ }^{1}$
}

\begin{abstract}
Fragmentalism was first introduced by Kit Fine in his 'Tense and Reality' (Modality and tense: philosophical papers, Oxford University Press, Oxford, pp 261-320, 2005). According to fragmentalism, reality is an inherently perspectival place that exhibits a fragmented structure. The current paper defends the fragmentalist interpretation of the special theory of relativity, which Fine briefly considers in his paper. The fragmentalist interpretation makes room for genuine facts regarding absolute simultaneity, duration and length. One might worry that positing such variant properties is a turn for the worse in terms of theoretical virtues because such properties are not involved in physical explanations and hence theoretically redundant. It will be argued that this is not right: if variant properties are indeed instantiated, they will also be involved in straightforward physical explanations and hence not explanatorily redundant. Hofweber and Lange, in their 'Fine's Fragmentalist Interpretation of Special Relativity' (Noûs 51:871-883, 2017), object that the fragmentalist interpretation is in tension with the right explanation of the Lorentz transformations. It will be argued that their objection targets an inessential aspect of the fragmentalist framework and fails to raise any serious problem for the fragmentalist interpretation of special relativity.
\end{abstract}

Keywords Fragmentalism - Special relativity · Frame-relative properties · Perspectival facts

Martin A. Lipman

m.a.lipman@gmail.com;

https://www.martinlipman.org

1 Institute for Philosophy, Leiden University, Nonnensteeg 1-3, 2311 BE Leiden, The Netherlands 
Consider some faraway place in the universe. There is no fact of the matter about what happens there at the very moment you are reading this sentence. It is a mistake to think that there is an objective course of time through which all current things in the universe are progressing. It is also not the case that the things you see have intrinsic mass, or intrinsic spatial shapes. These are some of the standardly accepted metaphysical consequences of the special theory of relativity, captured by the Minkowskian conception of spacetime.

The Minkowskian conception of spacetime accepts the assumption that what varies across perspectives (such as frames of references) must be mere appearance. This assumption is rejected by a metaphysical framework known as fragmentalism. Fragmentalism holds that what varies across perspectives are not mere appearance but genuine bits of reality. These perspectival facts are located in different fragments. The view was first introduced by Fine (2005). He argues that a fragmentalist version of tense realism is superior to more standard versions of tense realism. In particular, Fine argues that a fragmentalist view of time is in a position to (1) better account for the passage of time, (2) better account for the relation between tensed talk and temporal reality, and (3) that it renders tense realism compatible with (a fragmentalist interpretation of) the special theory of relativity.

The focus of this paper is not on the fragmentalist interpretation of special relativity as a way of bolstering a tense realist view of time. The focus of this paper will rather be on the interpretation of special relativity as such and explores its worth as a self-standing view. Of course, by showing how the fragmentalist interpretation of special relativity is independently motivated and not an ad-hoc reinterpretation, the current paper provides further support for Fine's overall argument. The main aim of this paper is however to provide further clarification of the fragmentalist approach to special relativity and to show that, though highly nonstandard, it is of intrinsic interest, regardless of one's views of tense realism.

Since Fine's paper, there have been various discussions of the conceptual foundations of fragmentalism and various alternative formulations have been offered (see Lipman 2015; Loss 2017; Simon forthcoming). The aim of the current paper is not concerned with the conceptual or technical aspects of fragmentalism and I will for the most part restrict the discussion to Fine's formulation of fragmentalism. However, there will be one point where a difference in approach will be relevant to our discussion.

It is also important to keep in mind that none of what follows is in any way intended to be a criticism of the standard Minkowskian interpretation. To show how the fragmentalist interpretation is motivated by various considerations is not the same as showing that it is also better than the standard interpretation. These two points should be distinguished. In my view, the question of which theory is ultimately better will depend crucially on wider theory integration, how well each interpretation generalizes and meshes with other scientific theories. This question lies beyond the scope of this paper. The aim here is to show the independent interest of the view, not its superiority.

As the main interest in fragmentalism lies within metaphysics, the paper assumes no real background knowledge in the relevant physics and some basic matters will be briefly explained afresh. Apologies for those who know these matters well. 
The paper proceeds as follows. The first section introduces fragmentalism. The second section explains how a fragmentalist interpretation deals with the sorts of scenarios that are typically invoked to illustrate how special relativity is in tension with our more ordinary ways of thinking about space and time. After discussing the motivations driving the fragmentalist interpretation, it will be shown how the variant properties naturally play explanatory roles and are not merely epiphenomenal additions to our metaphysics. The paper will then discuss an objection against the fragmentalist interpretation due to Hofweber and Lange (2017). They object that fragmentalism is in tension with the right explanation of why the Lorentz transformations hold. The final section replies to their objection.

\section{Fragmentalism}

Fragmentalism is the view that the world is inherently perspectival. It is standardly assumed that whenever we are dealing with conflicting perspectival representations of reality, there is always some kind of relativization to standpoints involved in the underlying facts and that it is only ever representations that are properly said to be perspectival, and not the world itself (see e.g. Moore 1997: Ch. 3). We standardly assume that we only ever have perspectival representations of a non-perspectival world. Fragmentalism denies this assumption, allowing that the world is itself an inherently perspectival place where facts do not simply obtain or fail to obtain, as we ordinarily assume, but where certain facts can obtain in the context of one set of facts and yet fail to obtain in the context of other sets of facts.

Fine's formulation of the view revolves around a primitive relation that he calls coherence. The overall collection of facts, 'über-reality', includes pairs of mutually incompatible facts. They are the facts that we would be tempted to describe as being somehow 'from conflicting perspectives' such as obtaining 'at different times' or 'within different frames of reference'. Fragmentalism denies this substitution of the apparent facts with more relational facts and instead accepts the incompatible facts as being all equally constitutive of the world. Instead of 'relativizing' facts in some way or another, we should recognize that some of the facts cohere and some do not. Given this set up, there are maximal coherent collections of facts, which Fine calls the 'fragments'. These play various theoretical roles within the fragmentalist picture, such as playing the role of times (2005: 308-310) and playing the role of the indices at which we evaluate the truth of ordinary utterances (2005: §9).

Fine's characterization of fragmentalism follows his more general methodological view that the intended formulation of many metaphysical views requires a distinction between what is merely the case and what is in reality the case (see his 2001: §8-10, 2005: §2). What is in reality the case is expressed using a sentential operator $\Re(\ldots)$, which is governed by various principles. A metaphysical realist position about the fact that $p$ should be understood, according to Fine, as the position that it is in reality the case that $p$, i.e. that it is the case that $\Re p$. Tenserealism, on this approach, becomes the claim that various tensed sentences are embedded under the reality operator $\Re$ in a correct description of reality. Which facts are real together and which are not is expressed using the earlier mentioned 
notion of coherence. One possible view here is that two facts are real together if it is in reality the case that they cohere. But Fine suggests that it might be possible to understand the fragmentalist's notion of reality in terms of coherence by assuming that a fact is real if and only if it self-coheres (2005: 281, fn.13). This way we do not need both the notions of reality and coherence, we can make do with the single notion of coherence-stipulated as itself conveying the fundamental reality of whatever it relates. To express that A and B 'cohere', we can just write that they are real together, i.e. that $\Re(\mathrm{A}, \mathrm{B})$.

The central thesis of fragmentalism is that conflicting matters that appear to obtain from equally good but conflicting perspectives, whether they be different times, or some other kind of 'perspective', do not have to be mere appearances of an underlying layer of compatible facts. In the case of the special theory of relativity, we encounter a particularly striking case of conflicting facts across perspectives. There are different possible accounts of what the relevant 'perspectives' are in this context. Einstein's (1905) so-called 'radar' definition of simultaneity uses a relativization to paths of inertial motion through spacetime; but one could also relativize to spacetime locations, or to pairs consisting of frames of references and times, what we may call 'frame-times' (Fine 2005: §11). Regardless of the exact nature of the index of relativization, these indices or 'perspectives' disagree about which events succeed one another, about the duration of a given event, and the intrinsic spatial shapes and intrinsic masses of objects. These are the so-called variant (or 'frame-relative') properties of special relativity. When ' $p$ ' is a sentence attributing some frame-relative property to an object or event, realism about such properties consists-on Fine's approach—in accepting that $\Re p$. The fragmentalist interpretation of the special theory of relativity requires not a revision of the way we think about space, time and their occupants, but requires rather a revision of the metaphysical structure we attribute to reality: it takes there to be various collections of cohering facts and, across such fragments, incompatible facts concerning the variant properties of things. As Fine remarks, the resulting view can be pictured as 'a plurality of physical space-times [...] each of them Newtonian in structure' (2005: 306). ${ }^{1}$ This should become clearer when we have a closer look at the application of fragmentalism to special relativity.

\footnotetext{
1 It is worth noting that there are other possible fragmentalist interpretations of special relativity, some of which stay much closer to the Minkowskian conception of spacetime. Stein (1968) mentions for example the possibility of a 'solipsist' interpretation according to which 'for any event, it and it alone is real' (1968: 18). This locational view can be captured in the fragmentalist framework as the view that only the properties of a single relativistic event all cohere. Fine (2005: 304-305) briefly argues against this view within the context of tense-realism, pointing out that a tense-realist aims to maintain a real difference between spatial and temporal indexicality, which is lost on this view. The following discussion is not meant to apply to all possible fragmentalist interpretations.
} 


\section{Fragmentalist relativity and the manifest image}

The Minkowskian conception effectively rejects the reality of anything that differs across the relativistic perspectives and only takes that which is invariant to be real. ${ }^{2}$ The result is the familiar fusion of space and time into a single spacetime, rejecting the various properties that are based on a neat separation of space and time, such as shape, duration, rest mass, simultaneity, and so on. These are now the 'mere shadows' of reality, as Minkowski (1964/1908: 297) puts it. The relation between the Minkowskian conception of spacetime and our more common understanding of our spatiotemporal surrounding can be neatly conveyed through various well-known scenarios involving different observers in relative motion to each other. To better see how the fragmentalist framework implies a more conservative relation between special relativity and our manifest image, it will be helpful to consider these wellknown scenarios afresh.

Imagine that you are in a space shuttle hanging still in an empty black space and that, for as long as you remember, you have experienced your space shuttle as being at rest. Imagine that I am in an exactly analogous situation, that is to say, I am also in a space shuttle that, for as long as I remember, I have experienced as being at rest. One day, we see each other's space shuttles. You experience my space shuttle as drifting by with a constant speed of $5 \mathrm{~km} / \mathrm{h}$ towards the east, still experiencing yourself at rest. I experience you as drifting by with a constant speed of $5 \mathrm{~km} / \mathrm{h}$ towards the west and still experience myself as being at rest. Our observations of each other's shuttles therefore disagree about who is moving and who is at rest: you observe that I am moving, whereas I observe myself as being at rest.

You set up an experiment. You are standing in the exact middle of your space shuttle (which you still observe as being at rest) and you emit some light towards both ends of the space shuttle. You observe that the light arrives simultaneously at the front and end of the shuttle. This is as you would expect: as you are at the middle of a space shuttle at rest, the light has the same distance to travel in both directions and should arrive at the same time, given that light always has the same speed $c$ (roughly 300,000 kilometres per second) regardless of what direction it moves in. I am in my space shuttle, observing your experiment. From my perspective, the beams of light also have the same velocity $c$ as in yours. But since I observe your shuttle as moving, the forward moving light has to travel more distance, and since it still has the same constant speed $c$, arrives later than the light sent towards the back. As before, our observations conflict. This time, our observations do not disagree about the constant velocity of light, but about the simultaneity of the light arriving

\footnotetext{
${ }^{2}$ Our discussion will employ only coordinate descriptions of the Minkowski spacetime instead of the more typical geometric description of spacetime as a 4-dimensional real vector space together with a certain metric signature (Naber 1988: 1). This characterization of the intrinsic geometric structure is not congenial to our discussion since it has already abstracted away the perspectival representations that are the common starting point with the fragmentalist. This paper is concerned precisely with the assumption at play in the transition from the perspectival representations to the intrinsic structure of spacetime and so Minkowski's own Erlanger approach of seeing intrinsic structure as that which is invariant across coordinatized descriptions provides a more suitable setting for our discussion.
} 
at, respectively, the front and back of your space shuttle: you observe them arriving simultaneously, whereas I observe them arriving one after the other.

This sort of scenario was originally told in terms of trains (Einstein 1920: Ch. IX; see also Einstein 1905: §A.2) and there are some complicating factors that we have glossed over. The crucial overall point however is that there is no way whatsoever of singling out one of the two conflicting sets of observations. Could one of us be right? According to Newton, only one of us is indeed right. Newton took space to be a three-dimensional spatial realm that endures through time, so that any spatial location at one time is taken to be identical to a spatial location at a different time, and which location is which is an entirely objective matter (Newton 1689/1934: 6). But how can we figure out which spatial location at one time is identical to which spatial location at a different time? It seems that we cannot tell empirically who is right.

The different locations do not come with intrinsic qualitative differences with which we could individuate them, telling us which point is which. The constant velocities of objects that we observe also cannot help arbitrate our dispute, given that our observations disagree precisely about which objects have which constant velocity. Properties other than the observed constant velocities do not help either. As Galileo famously showed, we cannot tell from properties other than their constant velocity whether a given object has a certain constant velocity, since things look exactly the same in scenarios seen from different frames of reference (Galileo 1632/1967: 186-187). In short, it does not help to appeal to the contents of our conflicting observations because our observations agree about everything except for the constant velocities attributed to things.

It seems we could only arbitrarily assign one of us to be the privileged observer, without any possible empirical evidence for any particular privileging. Moving from a Newtonian to a Minkowskian conception of spacetime eradicates the threat of empirical arbitrariness, as the Minkowskian view eliminates precisely that about which the different perspectival observations conflict. An important role here is played by the Lorentz transformations (together with other transformations, such as those governing electric fields) as they capture exactly what varies and what remains invariant across perspectives. Lorentz transformations are coordinate transformations between two inertial coordinate frames that move at a constant velocity relative to each other. If we only consider coordinate systems that are oriented the same way and which are such that one moves only in the $x$-direction of the other frame, the Lorentz transformations are the following (with the primed coordinates for the moving frame):

$$
\begin{aligned}
t^{\prime} & =\gamma\left(t-v x / c^{2}\right) \\
x^{\prime} & =\gamma(x-v t) \\
y^{\prime} & =y \\
z^{\prime} & =z
\end{aligned}
$$

The so-called spacetime interval between spacetime points is the central invariant property that constitutes the intrinsic structure of Minkowski spacetime. It is written 
$\Delta s^{2}$ and defined as follows: $\Delta s^{2}=(c \Delta t)^{2}-\Delta x^{2}-\Delta y^{2}-\Delta z^{2}$. Here $\Delta x, \Delta y, \Delta z$, and $\Delta t$ can each be different in different Lorentz-related frames but only in such a way that $\Delta s^{2}$ comes out the same in each frame description (where by 'frame description' I mean a description of events in terms of the coordinates given by a particular frame of reference). According to the Minkowskian interpretation, the spacetime interval $\Delta s^{2}$ is the only real spatiotemporal quantity, which can be broken up into different temporal and spatial components within different coordinate representations. The different temporal and spatial components only feature within the coordinate representations, which are now deemed to be the perspectival representations of the underlying non-perspectival world, itself only characterized by the spacetime interval.

The fragmentalist framework offers an alternative treatment of the shuttle scenario. Taken at face value, we have two conflicting descriptions that, together, imply incompatible assignments of variant properties. If we forget the Minkowski interpretation for a moment, it is an appealing thought that variant properties are instantiated out there. The very property of squareness studied in Euclidean geometry is plausibly thought to be the sort of property instantiated by the objects around me. This is how many of us understand the world in our less-guarded moments, indeed, we even take ourselves to observe such properties (see Epstein 2018; see also Chalmers 2012: Ch. 7 on 'Edenic squareness'). A piece of paper can be square according to descriptions in one frame of reference and oblong according to descriptions in another frame of reference. We expect that there is a stark difference in what it is like to undergo an experience of a square shape and undergoing the experience of an oblong shape, and this phenomenal difference seems due to a difference in content: they would be experiences that attribute different shape properties to things, the very shape properties for which there is no place in the Minkowskian conception of the world (cf. Siegel 2010: Ch. 3).

If the Minkowskian interpretation is true, these assumptions about our observations are controversial. If the variant properties are not instantiated out there, then externalist considerations about the contents of perception might lead us to think that such properties also cannot really feature in the representational contents of our perceptions and observations (Chalmers 2012: Ch. 7). But this is just to say that if the Minkowskian interpretation is right, we also need to revise our ordinary assumptions about the sorts of properties that we take ourselves to observe. The fragmentalist interpretation has appeal because it restores a simple story about what sort of properties things have, and hence how we perceive the world to be. It offers an alternative interpretation on which the intuitive story of the apparent contents of our perceptual experience can be right.

Consider in a little more detail the conflicting observations we encounter in the space shuttle scenario. I have no good independent reason to discredit your observation, and you do not a have good independent reason to discredit my observation. Between us, it seems that we have equally good evidence that I am at rest as well as evidence that I have a constant velocity. In the imagined case, you experience my space shuttle as moving and your space shuttle as being at rest; I experience your space shuttle as moving and my shuttle being at rest. Given these 
observations, a simple fragmentalist description of the case imagined looks as follows ${ }^{3}$ :

$\Re$ (your shuttle is at rest, my shuttle moves with $5 \mathrm{~km} / \mathrm{h}$ to the east, the light beams arrive at the front and back simultaneously) \& $\Re$ (your shuttle moves with $5 \mathrm{~km} / \mathrm{h}$ to the west, my shuttle is at rest, the light arrives at the front only after it arrived at the back)

Neither of our observations is arbitrarily privileged according to these descriptions. We take what we observe, namely the co-instantiation of various variant properties, at face value. We accept that your shuttle is at rest and that your shuttle is moving with a constant velocity; and we accept that the light arrives at the front and back simultaneously and that it arrives at the front only after it arrived at the back. More generally, we both observe genuine but conflicting facts concerning the simultaneity, duration, length and shape. The discussed sort of scenario thus offers support for a fragmentalist conception that matches the overall pool of naïve observation reports whilst taking the content of these descriptions at face value.

One might worry that the fragmentalist picture not only opposes the Minkowskian view but more generally opposes an instance of so-called symmetry reasoning. The symmetries of theories, in this case the re-descriptions of facts in different frames of reference related by the Lorentz transformations, are normally treated as a guide to reality: we consider the variance of properties across such frame descriptions to be a sign of their unreality (see, amongst others, Baker 2010; Dasgupta 2016; Earman 1989; Ismael and van Fraassen 2003; and Roberts 2008). One might worry that fragmentalism conflicts with this widely adopted method of symmetry reasoning. But this is not quite right. The relevant descriptions in cases of symmetry reasoning are all descriptions that are meant to be equally good complete descriptions, of all the facts. Treating invariance amongst arbitrary incomplete representations as a guide to reality would be quite disastrous, as any unmentioned feature would be filtered out in this way. On the other hand, if the varying descriptions are indeed equally good complete descriptions, then it simply follows that any remaining differences amongst them must indeed be merely conventional differences in the ways we describe the world, otherwise any such description would not be complete.

For the fragmentalist, a complete description of some physical system is a description of the system in all the different fragments, of its total perspectival manifestation. We should sharply distinguish between what is variant across the frame descriptions within a single collection (i.e. across fragments) and what is variant across the total collection of such descriptions (i.e. across total representations of the overall fragmented world). Even on the fragmentalist view, there is the standardly presumed connection between reality and invariance across equally good complete representations of some isolated system. The fragmentalist is not denying this connection between variance amongst complete descriptions and mere ways of

\footnotetext{
3 This is not quite the description that Fine would give. For reasons that we cannot go into, Fine believes that the embedded tensed sentences are not of the simple form 'the shuttle is at rest' but rather 'a token fact of the shuttle's being at rest obtains' (2005: 318).
} 
describing things, the fragmentalist only denies that descriptions of single fragments are complete descriptions and hence denies that the variance across fragments is an instance of variance across equally good complete descriptions. Fragmentalism does not oppose standard symmetry reasoning. It only implies that the reasoning does not apply in a case where it is standardly taken to apply. I will continue to speak of frame-invariant facts and variant properties, even within the fragmentalist picture; I hope I have said enough to avoid confusion.

\section{The explanatory potency of variant properties}

Giving that the standard Minkowskian interpretation of the special theory of relativity is both successful and explains matters in terms of invariant properties only, does the fragmentalist not introduce a layer of superfluous qualitative structure, unneeded for the scientific explanation of phenomena? If so, parsimony considerations will speak strongly against a fragmentalist interpretation. Contrary to the assumption behind this worry, it turns out that variant properties will naturally slot into various kinds of physical explanations, due to the fact that they would themselves exhibit lawlike dynamic behaviour.

The variant properties, if they are indeed instantiated, will themselves be amongst the properties that are governed by dynamic laws. The variant properties of things at one time naturally figure in explanations of variant properties at a later time and so they are not explanatorily redundant. Given that the attribution of variant properties differs across fragments, and given that dynamic explanations feature such properties, one and the same phenomenon may be explained in quite different ways in different fragments.

This is nicely illustrated by the following well-known case (taken from Mermin 2005: 185-186 but originally due to Bell 1976/1987). Imagine that there are two rockets Rocket I and Rocket II, separated by some spatial distance $\Delta x$, with a rope stretched tightly between them. Say we observe things as follows: Rocket I and Rocket II are initially at rest and then they start to move in the $x$ direction at the same time at the same rate, keeping their spatial separation $\Delta x$ unchanged. As the rope has gained at this later moment in time a velocity in the $x$ direction, the length of the tightly strung rope must be contracted. Now imagine that the rope is contracted beyond its elastic limit and breaks at the moment that the rockets are moving. Here we explain the breaking of the rope in terms of the contracted length of the rope and the constant spatial separation between Rocket I and Rocket II. We thus have an explanation in terms of length contraction.

Things are different from the perspective of a different frame: here Rocket I and Rocket II are initially moving in the $-x$ direction and then come to a standstill at which moment the rope breaks. (From our earlier perspective, we say that this is the perspective of someone initially moving in the $x$ direction). It can be shown that the moment that Rocket II stops comes before the moment that Rocket I stops, so that Rocket I continues in the meantime to travel in the $-x$ direction, thereby stretching the rope until it breaks. Here we explain the breaking of the rope in terms of a temporary difference in constant velocities between Rocket I and Rocket II, and the 
briefly increasing distance between them. As Mermin points out, 'the mechanism that gives the real explanation for a phenomenon in one frame of reference, may be quite different from the mechanism that gives the real explanation in another' (2005: 185).

There will be fragment-specific phenomena which can only gain fragmentspecific explanations. To take another simpler case: constant velocities at one time are naturally involved in explanations of constant velocities at a later time (cf. Sklar 1977: 180, Dasgupta 2016: 846). If in one fragment an object remains at rest, its being at rest at $t_{1}$ may be explained by it having being at rest at $t_{0}$ and its having being unperturbed by external forces in the time between $t_{0}$ and $t_{1}$. Given that the object's being at rest is unique to the relevant fragment, it also only receives an explanation within this fragment. ${ }^{4}$

The variant properties are certainly not physically redundant within the fragmentalist conception of space and time, and feature in physical explanations of phenomena. This can seem puzzling: surely the Minkowski conception, which enjoys such wide adherence, is not lacking in adequate physical explanations and yet it does not recognize the instantiation of variant properties, nor explanations phrased in terms of them. The Minkowskian is indeed not lacking in adequate explanations, but that is because the Minkowskian does not acknowledge the phenomena that would stand in need of explanations in terms of variant properties. Because the Minkowskian denies that an object is really at rest at a given time for example, she also does not need to explain this in terms of changes in the constant velocity of the object at a previous time. The Minkowskian only acknowledges phenomena characterized in terms of spacetime intervals, and she also only needs to appeal to (changes in) spacetime intervals to explain them. The Minkowskian is thus not lacking physical explanations when considered on its own terms.

The fragmentalist and Minkowskian differ over what the phenomena are to which our physics should be held accountable. The fragmentalist admits a richer variation of phenomena, for example, it distinguishes a case in which two particles are both at rest from a case in which two particles are both travelling with $5 \mathrm{~km} / \mathrm{h}$ in the $x$ direction. If there are indeed such phenomena, it is not just that variant properties happen to be serviceable in physical explanations, they serve in physical explanations that could not be given in any other terms. The main issue is thus whether we should construe the phenomena-the legitimate explananda-as including perspectival matters or not.

\section{Hofweber and Lange on the fragmentalist interpretation}

Hofweber and Lange argue that the fragmentalist interpretation of special relativity is in tension with the proper explanation of why the Lorentz transformations hold (2017: 871). On the standard interpretation, the Lorentz transformations of the

\footnotetext{
${ }^{4}$ Cf. Sklar: 'As von Neumann has remarked, the problem with a non-relativistic explanation of the facts is not that one can't be given but that too many can be given, and no reason can be given for selecting one rather than another' (1977: 280).
} 
different frame descriptions obtain because of the way in which the same underlying world is coordinatized in the different frames of reference. Hofweber and Lange argue that this is the right explanation of why the Lorentz transformations hold and that the fragmentalist has no room for this explanation.

One preliminary point. I do not agree with the theoretical commitments attributed to the fragmentalist interpretation in their discussion. Hofweber and Lange assume that fragmentalism 'takes frame-dependent facts to be fundamental rather than derivative from frame-invariant facts such as facts about the spacetime intervals between various events' (2017: 874). But nothing that we have seen so far commits the fragmentalist to this. For one, Fine himself is not committed to the idea that something is in reality the case if and only if it is not grounded by anything, so two matters may both be real even when the one grounds the other (2001: 27). Fine's notion of 'reality' is independent from 'grounding'. Although grounding may be a good defeasible guide to what is real, there is no reason why there could not in principle be some fundamental facts grounding certain other fundamental facts (presumably, with the relevant grounding facts themselves being fundamental as well).

In other words, there is nothing in the fragmentalist interpretation that is incompatible with postulating a Minkowskian spacetime next to the various fragments and taking the distributions of the variant properties to be grounded in the spacetime intervals of the Minkowskian spacetime. Such a view does not disagree with the standard Minkowskian interpretation about the grounds or the explanations of the variant matters. The Minkowskian takes them to be explanations of mere appearances, whereas the fragmentalist takes them to be explanations of genuinely obtaining yet grounded facts. Such a view would only disagree with the Minkowskian view about the reality of the variant matters.

One might wonder whether there is still a substantive difference between the Minkowskian view and the current way of developing the fragmentalist account. What it is the theoretical upshot of the variant matters being 'real' in Fine's sense? The importance is that of marking a metaphysical realism about those variant matters. The relevant question is whether realism or antirealism is true about the frame-relative facts, that is, whether consideration of the special theory of relativity removes all frame-relative facts from one's metaphysical conception of reality: the Minkowskian answers yes, the fragmentalist answers no. There is of course a further question whether realism is best understood using Fine's primitive notion of reality (see Horwich 2007; to which Fine 2007 responds; see also Lipman 2018).

As mentioned briefly in the introduction, alternative formulations of fragmentalism, given by Lipman (2015), Loss (2017) and Simon (forthcoming), do not invoke a primitive notion of 'reality' but rather only work with the notion of coherence, which they prefer to call 'co-obtainment'. Two facts may obtain without co-obtaining and this is what the 'fragmentation' consists in. On these views, the debate with the Minkowskian is not whether the frame-relative facts are fundamental or real facts, as on Fine's approach, rather, the debate is about whether they are facts at all. The fragmentalist believes that it can be a genuine fact that a piece of paper is square and a genuine fact that it is oblong, whereas the Minkowskian needs to say that the piece of paper does not have such a property but 
only seems to, given a certain frame-relative description. If grounding is assumed to be factive, the Minkowskian cannot be claiming that the frame-relative facts are themselves grounded in the spacetime interval (i.e. they cannot claim that it is the paper's being square that is grounded in the interval), as this would imply that there are genuine frame-relative facts (i.e. that the paper is genuinely square and hence that there is the purely spatial property of being square). If there are such genuine frame-relative facts, this raises the question whether we should privilege one frame or accept the mutually incompatible facts of multiple frames. Neither of these options are typically taken to be live options for the Minkowskian view. Instead, the Minkowskian must be saying that, strictly speaking, it is only the mere appearance of frame-relative facts that is grounded in spacetime intervals. Though the Minkowskian and the current type of fragmentalist disagree about the exact nature of the explananda, they agree about the standard direction of explanation, both taking the spacetime interval to ground the variant matters (or, according to the Minkowskian, the appearances thereof).

The complaint that the fragmentalist interpretation conflicts with the right explanations of the Lorentz transformations is therefore somewhat off the mark: the objection targets something that is not really an essential commitment of the view, namely a claim about the direction of explanation. The fragmentalist can in principle adopt whatever direction of explanation the Minkowskian appeals to.

Having said this, Hofweber and Lange's objection is interesting and it is worth considering the sort of fragmentalist view it does target. Let us therefore imagine a fragmentalist who believes: (1) that the variant properties are real, and (2) that the spacetime intervals consist in constant ratios of variant properties, and (3) that the variant properties - the different spatial and temporal distances in different frames-ground such ratios. The invariant intervals are the unified appearances of a fundamentally fragmented world on this way of developing the fragmentalist interpretation. The Lorentz transformations of the coordinate frames underwrite the law-governed regularity between the distributions of fundamental yet variant properties in the different fragments. It is this sort of view that Hofweber and Lange criticize and it is a grounding story that might indeed appeal to the fragmentalist. For the remainder of this section, the fragmentalist interpretation under discussion will be the one just outlined.

Both the Minkowskian and the fragmentalist agree that the Lorentz transformations hold and that the spacetime interval is invariant. Hofweber and Lange claim that the invariance of the interval is generally assumed to explain the Lorentz transformations (2017: 876). The assumed explanatory priority of the spacetime interval, they argue, is naturally explained by the standard interpretation of the special theory of relativity:

Why does the spacetime interval's invariance count as explanatorily prior to various other facts, such as the transformation laws? After all, the transformation laws suffice to entail the spacetime interval's invariance. Why does science take the direction of explanation as running from the spacetime interval's invariance to the transformation laws rather than, say, in the reverse direction? Because the spacetime interval, as a frame-invariant fact, is the 
reality, whereas the facts related by the coordinate transformations are framedependent facts and hence are appearances of that reality. How things are explains how things appear from a given perspective. Therefore, the law that a certain quantity is invariant takes explanatory priority over the laws specifying how various frame-dependent quantities transform (2017: 876).

Starting from the Lorentz transformations plus supplementary assumptions, we can derive the invariance of the spacetime interval. In the other direction, so starting from the invariance of the spacetime interval plus supplementary assumptions, we can derive that the Lorentz transformations hold. About all of this, the Minkowskian and fragmentalist can agree.

Nevertheless, it is standardly assumed that the explanatory direction runs from the invariance of the spacetime interval to the Lorentz transformations. Hofweber and Lange suggest in the cited passage that this is because the spacetime interval is real and the variant properties mere appearances. The fragmentalist interpretation under discussion, which takes the variant properties to be real and the grounds for the spacetime interval, cannot offer the standard explanation. The standard interpretation underwrites the standardly assumed explanatory priority of the spacetime interval, whereas the fragmentalist conflicts with it.

It is unclear what the exact dialectical significance is of this point. We can discern two distinct criticisms. First there is the claim that the invariance of the interval is widely assumed to have explanatory priority within the scientific community. This should be distinguished from the claim that the invariance of the interval indeed has explanatory priority. The first is a sociological claim about, roughly, the assumptions at play in the scientific community; the second is a claim about which fact indeed takes explanatory priority. Let us consider these points in turn.

First there is the claim that the invariance of the spacetime interval is generally assumed to take explanatory priority. Hofweber and Lange offer various quotes (by Eddington, Mermin, Einstein and Holton) which express adherence either to the standard interpretation or to the basic assumption that different perspectives always present us with mere appearances of an underlying unified reality (2017: 874). This assumption —of taking reality to be unified behind the mere perspectival appearances-they claim is 'alien to the spirit behind fragmentalism' (2017: 877). Let us grant that this is so. What to make of this? One might here raise the methodological principle that in any disagreement between the views of scientists and metaphysicians, we ought to favour the views of the scientists, given that science is on a much firmer epistemological footing. Though we should all adhere to this methodological principle, it has to be wielded with care. The scientist's views are authoritative because the scientific method has proven to be the best means of furthering knowledge. The epistemological deference to science is based on the methodological superiority of the scientific method, and hence the principle of 'favoring the scientist's views' should clearly be restricted to only those matters that can be decided on the basis of those scientific methods - that is, to the empirical claims. The scientific method only provides the scientist with an authority over empirical matters. The fragmentalist does not question the special theory of relativity, the 
transformation laws, or any of the empirical evidence that supports them. There is no disagreement about the science itself. The debate is about the proper metaphysical interpretation of the relevant science: about how best to conceive of the world so as to accommodate the scientific facts. This is not decided by the standard empirical methods (if only we could), but on the basis of simplicity, plausibility, the ability to integrate with further theories, the ability to save the appearances, and so on. These methods are not a firmer footing than those of the metaphysician, in fact they arguably are amongst the methods of the metaphysician. There is no good basis for deferring to the views of scientists on these abstract and non-empirical matters.

Against this response, one might argue that we ought to adopt a more farreaching naturalism on which we philosophers ought to adopt whatever views are congenial to current scientific practice, even on matters that lie beyond empirical matters. Physicists explain variant matters on the basis of invariant matters, and would it not obstruct science if fragmentalism demands a change in the current scientific practice $?^{5}$ Ultimately, current practice ought to conform to whatever turns out to be the most reasonable overall view of the world. The sensible fragmentalist metaphysician is not currently suggesting to overthrow current scientific practice, she is just showing that there is a coherent alternative to the standard interpretation and exploring its advantages. If the case for the standard interpretation remains stronger than the fragmentalist alternative, then this is reason enough to dismiss the fragmentalist interpretation. If there turn out to be major advantages to the fragmentalist interpretation over the standard interpretation, then it also would not obstruct science to change current practice, on the contrary. Either way, appeal to scientific practice at the current stage of investigation strikes me as a red herring.

There is another way of understanding the complaint that fragmentalism goes against general assumptions of the scientific community. The point may simply be that fragmentalism is a revisionary view and that this counts in favour of the standard interpretation, as the standard view has earned its credits and demonstrated it can provide an understanding of matters. Fragmentalism is indeed a revisionary view and this is indeed a comparative cost. This goes without saying. Further work will clarify how revisionary it is and whether advantages offset it. There are also distinctions to be made. The fragmentalist approach sketched earlier, which maintains explanations in terms of a fundamental Minkowskian spacetime, is less revisionary than the fragmentalist view currently under discussion, which also reverses the order of explanation. This may be a point in favour of the weaker approach, but not nearly a decisive point.

Let us turn instead to the endorsement of the standard explanatory direction. Hofweber and Lange claim that the standard assumption is right and that the invariance of the interval has indeed explanatory priority. ${ }^{6}$ They write that the interval must be what explains Lorentz transformations 'because the spacetime

\footnotetext{
5 Thanks to an anonymous referee for pressing the naturalist underpinnings of the objection.

${ }^{6}$ I assume that the notion of explanation here is not merely epistemological, but something that is meant to reflect a certain objective explanatory order in the world.
} 
interval, as a frame-invariant fact, is the reality, whereas the facts related by the coordinate transformations are frame-dependent facts and hence are appearances of that reality' (2017: 876). This is not an argument but the direct denial of fragmentalism, which just is the view that the frame-relative facts are real and not the mere appearances of frame-invariant facts.

Hofweber and Lange argue that the fragmentalist needs to postulate 'brute' connections across the fragments without giving any reason to expect such brute connections, and that this is a substantive cost of the view (2017: 878). The relevant question here, I take it, is this: why are there only ever fragments related to each other in accord with the Lorentz transformations, and no other fragments? The answer is that there are the Lorentz 'laws' relating the fragments. There is no further explanation of why these laws hold. The Minkowskian, in contrast, explains the Lorentz transformations, according to this objection.

That the lawlike-connections underwriting the Lorentz transformations take explanatory priority over the spacetime interval (and assumed variance of the variant properties) does not mean that there cannot be another explanation of the Lorentz transformations. One might offer an account along general Humean lines, for example, or one might endorse a so-called dynamical explanation of the Lorentz transformations, according to which transformations are taken to be grounded in the various forces acting on rods and clocks (Bell 1976/1987; Brown and Pooley 2006; and Brown 2005). Such explanations are not incompatible with fragmentalism (or the theory of special relativity, more generally).

If such explanations are not to be had, however, then there will indeed be a brute constraint on the distribution of variant properties, but if this is how things turn out, this type of brute constraint arises in some form for any view that does justice to the special theory of relativity. The constraint that the fragmentalist needs to impose on the fragments is effectively that light must have the constant velocity $c$, regardless of the state of motion of the emitting body, in any fragment. This is just the postulate of the invariant velocity of light. The Minkowskian captures this invariance of the speed of light in inertial reference frames in terms of the geometric structure imposed on spacetime (together with the way this is projected onto inertial frames). But that this constraint is incorporated as a geometric law of spacetime does not mean that it is somehow less brute. Why does spacetime have the Minkowski structure as opposed to a geometric structure that allows objects to move faster than the speed of light? The Minkowskian answer is that it just does. This is entirely fair. The fragmentalist's postulation of the invariance of the velocity of light as a basic physical law is however no less fair.

One might think that the worry concerns not the brute imposition of the invariance of the speed of light, but rather the fact that the Minkowskian can explain why events have the variant properties that are attributed to them in a given frame. The Minkowskian explains variant properties in terms of the intervals of those events. The fragmentalist view under discussion takes the instantiations of the variant properties to be basic, and not to be the mere appearance of underlying properties. But that is only half of the story. This type of fragmentalism was characterized by the further idea that we can explain why the events have the spacetime intervals in terms of the variant properties of those events, in contrast to 
the Minkowskian view, on which the distribution of spacetime intervals are taken to be basic. Where two views differ in what grounds what, bruteness will emerge in different places. In such cases, our evaluation goes astray if we merely point out that a certain matter is brute on one view and not brute on the other.

Hofweber and Lange do not succeed in raising any serious problem for the fragmentalist interpretation of special relativity. It remains a live option, worthy of further investigation.

Acknowledgements This paper was written with support from the Humboldt Foundation (Kit Fine's Anneliese Maier Award) and substantially rewritten with support from the Netherlands Organization for Scientific Research (project number 275-20-055 - A Fragmented World). The material was presented at the Spacetime and Fundamentality conference at the University of Neuchâtel and at the Forschungskolloquium at the University of Hamburg. Many thanks to the audiences present at these occasions for their feedback. Many thanks also to the anonymous referees that have commented on this paper.

Open Access This article is distributed under the terms of the Creative Commons Attribution 4.0 International License (http://creativecommons.org/licenses/by/4.0/), which permits unrestricted use, distribution, and reproduction in any medium, provided you give appropriate credit to the original author(s) and the source, provide a link to the Creative Commons license, and indicate if changes were made.

\section{References}

Baker, D. (2010). Symmetry and the metaphysics of physics. Philosophy Compass, 5, 1157-1166.

Bell, J. S. (1976/1987). How to teach special relativity. In J. S. Bell (Ed.), Speakable and unspeakable in quantum mechanics. Cambridge: Cambridge University Press.

Brown, H. R. (2005). Physical relativity: Space-time structure from a dynamical perspective. Oxford: Oxford University Press.

Brown, H. R., \& Pooley, O. (2006). Minkowski space-time: A glorious non-entity. In D. Dieks (Ed.), The ontology of spacetime (pp. 67-89). Amsterdam: Elsevier.

Chalmers, D. (2012). Constructing the world. Oxford: Oxford University Press.

Dasgupta, S. (2016). Symmetry as an epistemic notion (twice over). The British Journal for the Philosophy of Science, 67, 837-878.

Earman, J. (1989). World enough and space-time: Absolute versus relational theories of space and time. Cambridge, MA: MIT Press.

Einstein, A. and (transl.) Beck, A., and (ed.) Stachel, J. (1905/1986). On the electrodynamics of moving bodies. In The collected papers of Albert Einstein, volume 2, supplement (pp. 140-171). Princeton: Princeton University Press.

Einstein, A. and (transl.) Lawsom, R. W. (1920). Relativity: The special and general theory. New York: Henry Holt Company.

Epstein, P. F. (2018). Shape perception in a relativistic universe. Mind, 127(506), 339-379.

Fine, K. (2001). The question of realism. Philosopher's Imprint, 1, 1-30.

Fine, K. (Ed.). (2005). Tense and reality. In Modality and tense: Philosophical papers (pp. 261-320). Oxford: Oxford University Press.

Fine, K. (2007). Response to Paul Horwich. Dialectica, 61(1), 17-23.

Galileo, D. S. (ed.) (1632/1967): Dialogue concerning the two chief world systems. Berkeley: University of California Press.

Hofweber, T., \& Lange, M. (2017). Fine's fragmentalist interpretation of special relativity. Noûs, 51(4), $871-883$.

Horwich, P. (2007). The quest for reality. Dialectica, 61(1), 5-16. 
Ismael, J., \& van Fraassen, B. (2003). Symmetry as a guide to superfluous theoretical structure. In K. Brading \& E. Castellani (Eds.), Symmetries in physics: Philosophical reflections (pp. 371-392). Cambridge: Cambridge University Press.

Lipman, M. A. (2015). On Fine's fragmentalism. Philosophical Studies, 172(12), 3119-3133.

Lipman, M. A. (2018). Against fundamentality-based metaphysics. Noûs, 52(3), 587-610.

Loss, R. (2017). Fine's McTaggart: Reloaded. Manuscrito: Revista Internacional de Filosofía, 40(1), 209-239.

Mermin, N. D. (2005). It's about time. Princeton: Princeton University Press.

Minkowski, H. (1964/1908). Space and time. In J. J. C. Smart (ed.), Problems of space and time (pp. 297-312). New York: Macmillian Company.

Moore, A. (1997). Points of view. Oxford: Oxford University Press.

Naber, G. (1988). Spacetime and singularities: An introduction. Cambridge: Cambridge University Press.

Newton, I., and (transl.) Motte, A. and (ed.), Cajori, F. (1689/1934). Philosophiae naturalis principia mathematica. Berkeley: University of California Press.

Roberts, J. (2008). A puzzle about laws, symmetries, and measurability. The British Journal for the Philosophy of Science, 59, 143-168.

Siegel, S. (2010). The contents of visual experience. Oxford: Oxford University Press.

Simon, J. (forthcoming). Fragmenting the waves. In K. Bennett \& D. W. Zimmerman (Eds.), Oxford studies in metaphysics: Volume 11. Oxford: Oxford University Press.

Sklar, L. (1977). Space, time and spacetime. Berkeley: University of California Press.

Stein, H. (1968). On Einstein-Minkowski space-time. Journal of Philosophy, 65(1), 5-23. 